\title{
A Microcontroller-Based 3-Axis Manipulator Applied to a Printed-Circuit-Board Driller System
}

\author{
Salvador Ramírez Zavala \\ Edmundo Barrera Cardiel \\ División de Estudios de Posgrado de la Facultad de Ingeniería Eléctrica \\ Universidad Michoacana de San Nicolás de Hidalgo \\ Av. Francisco J. Mújica s/n Ciudad Universitaria \\ Morelia, Michoacan, Mexico.
}

\begin{abstract}
This paper presents the design and construction of a 3-axis manipulator. The paper shows the application of the manipulator to a printed-circuit-board driller system. The manipulator uses direct current motors with permanent magnets. The design of the manipulator is based on microcontrollers to control the speed and position of the motors by using full bridge dc-dc converters with a pulse-width-modulation technique. The designed manipulator includes a personal computer (PC) that works as a user interface to load the file with the numeric control format for the driller. From this information, the PC generates the required position and speed commands for each servomechanism. The paper describes in detail the software and hardware designed to measure and control the speed and the position of each servomechanism.
\end{abstract}

Key-Words : - Robotics, Manipulators, Movement control.

\section{Introduction}

Manipulators have a broad application in industry. This paper presents the design and construction of a 3-axis manipulator. Experimentally, this manipulator has been applied in an ultrasonic testing system, to position the ultrasonic emiter/sensor. It has also been applied in a system to cut pieces with special shapes. In this application, it is required to control the orientation of the tool. This paper shows the application of the manipulator in a printed-circuitboard driller system.

The designed manipulator is a cartesian manipulator (X-Y plane) [1]. The drill (tool) is mounted on the $\mathrm{Z}$ axis. The motor drives are based on $\mathrm{dc}$ motors with permanent magnets, which were selected because of their high starting torque, low initial cost, and excellent drive performance [2]. The manipulator control system has a distributed structure. It has an independent control system for each servomechanism. The control of each servomechanism is based on a microcontroller. The power to each dc motor is supplied by a full bridge dc-dc electronic converter. A pulse-width-modulation (PWM) control method is applied. To measure the speed and position, a 256-slit optical encoder is mounted on the shaft of each motor. The following sections presents the details of the hardware and software designed for the manipulator.

\section{System Structure}

Fig. 1 shows a photograph of the printed-circuitboard driller system designed. The block diagram in Fig. 2 shows the general structure of the manipulator. It can be seen that the control system has a distributed structure. A brief description of the functions of each of these components follows:

The Personal Computer is used as the master controller of the manipulator. The PC calculates and sends the position and speed commands to each motor drive. These commands are calculated from a file with numeric control information. These files are obtained from a printed-circuit design program, such as Orcad, and have the following structure:

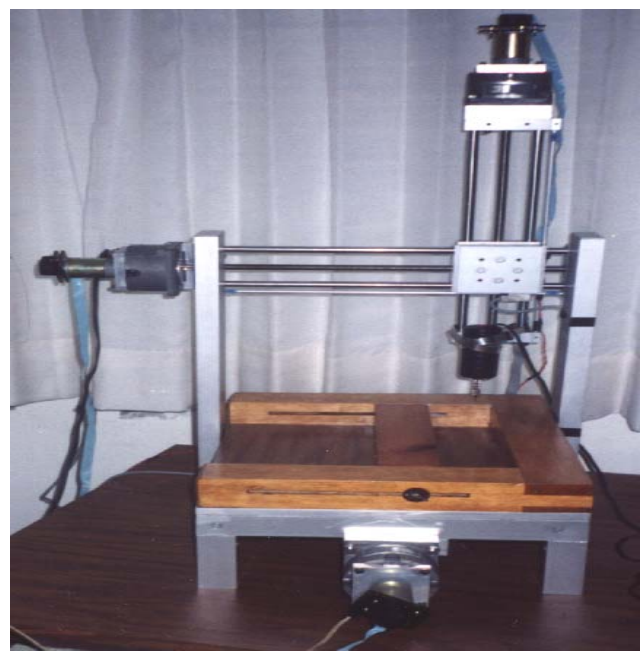

Fig. 1. Photograph of the manipulator 


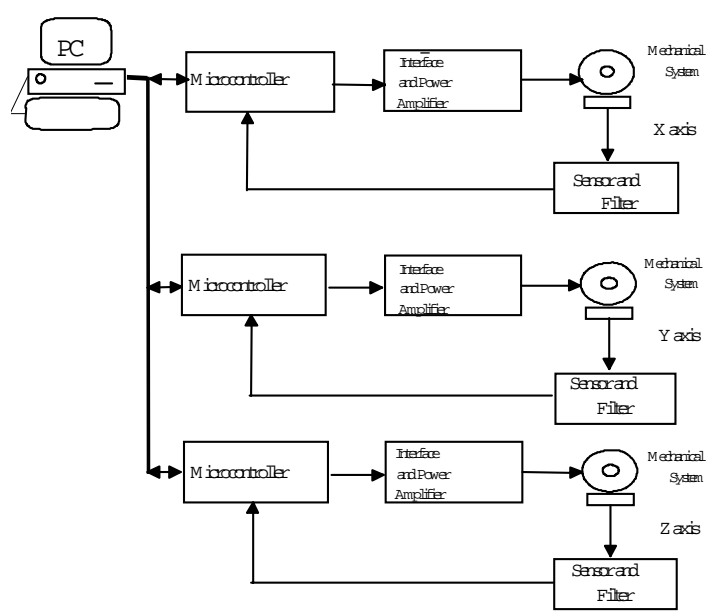

Fig. 2. General structure of the manipulator

\section{ASCII(bit size, position $x(1)$,position y(1))}

\section{ASCII(bit size, position $x(n)$, position $y(n)$ )}

The PC calculates the speed of each axis and synchronizes their displacements. To facilitate the operation of the driller system, a graphical user interface was designed. Fig. 3 shows this user interface. By means of this interface, the user can load the printed circuit file, can see the plot of the corresponding printed circuit, and can control the drilling process.

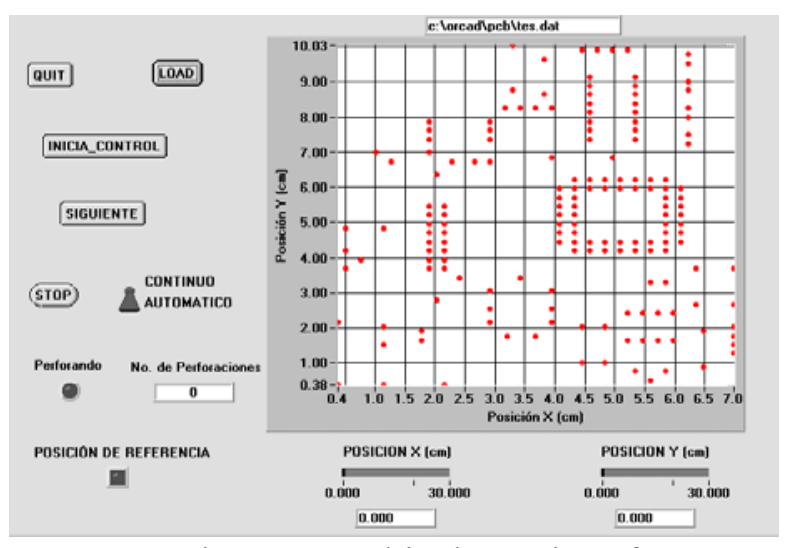

Fig. 3. Graphical user interface

The microcontroller used for the control of each motor driver is the Intel's $80 \mathrm{C} 196 \mathrm{KC}$ microcontroller [3]. Once the microcontroller receives the position, speed, and synchronization commands from the PC, it senses the position of the motor's shaft and calculates its speed.
Based on this information, the microcontroller calculates the speed control action. This digital control action is sent to the power amplifier module.

For the measurement of the position and speed of the motor, the microcontroller's High Speed Input (HSI) Unit is used. This unit can capture events with a $1 \mu$ s resolution in each of its four inputs (HSI.0HSI.3). In this design, we used two high speed inputs to measure de position of the motor's shaft (channels $\mathrm{A}$ and $\mathrm{B}$ of the encoder). The third high speed input is used to detect the index signal of the encoder. The fourth high speed input is used to detect the zero reference position of the corresponding axis.

The Interface and Power Electronics Converter changes the digital control action sent by the microcontroller into a voltage signal for the corresponding $\mathrm{dc}$ motor. Basically, the interface consists of a digital to analog converter and a PWM signal generator. The power electronic converter is a full bridge dc-dc converter built with MOSFETs.

The Mechanical System consists of a dc motor, a gear mechanism, a feed screw, and the load to be moved. In the $\mathrm{Z}$ axis, the mechanical system includes the tool, i.e., the drill.

The Sensor and Filter provide the microcontroller with a feedback digital signal of the position of the motor shaft. The sensor is a Hewlett Packard HEDS5000 encoder which has a disk with 256 slits. The encoder generates two pulse trains (channels $\mathrm{A}$ and B) that are in quadrature, and another pulse train called index that has a frequency of 1 pulse per revolution. A special filter, based on digital circuits, is used to eliminate possible "bounces" of the encoder signal [4].

\section{Position Control Algorithm}

The objective of the control algorithm is to generate the control action signals such that the manipulator follows the positions of the desired perforations. This objective can be reached if each servomechanism has a good position control of its motor's shaft. To control the position of each axis, the speed profile shown in Fig. 4 was selected [5]. 


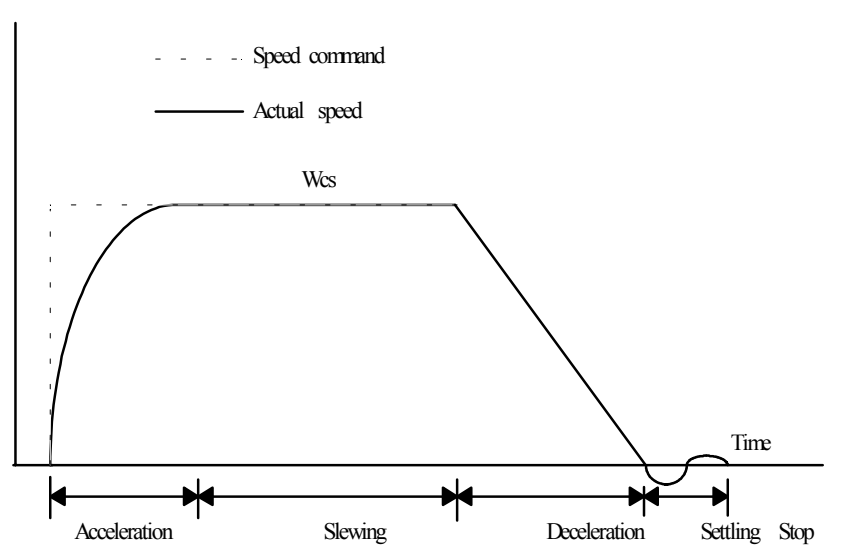

Fig. 4. Speed profile

In this profile, we can observe the following regions:

(a)Acceleration: The position error counter sends a command for maximum speed. The rotor is accelerated according to the dynamic equation of the motor and the speed controller used.

(b)Slewing: When the rotor reaches the desired maximum speed, the speed controller maintains this speed constant.

(c) Deceleration: To decelerate the rotor with a constant torque, the command speed $\omega_{\text {ref }}$ is given according with (1).

$$
\omega_{r e f}=\sqrt{2 \beta q}
$$

where:

$$
\begin{aligned}
& q=\text { actual error position } \\
& \beta=\text { rate of deceleration }
\end{aligned}
$$

(d) Settling: Ideally, the motor must stop at the target position and must not travel any farther when control is governed exactly by (1). In practice, however, there is always a possibility that the rotor overruns beyond the target, because the speed command data are not given as an ideal linear function but discontinuously owing to digital quantization. Normally, the system is designed so that the rotor eventually settles after several cycles of oscillation around the target. (e) Stop: When the rotor comes to rest at the target, the controller sends the required torque to maintain the rotor at that position. To get the speed profile shown in Fig. 4, the controller generates speed commands $\omega_{\text {ref }}$ that depend on the error position, according to the following rules:

i) if $0<q<q_{f} \longrightarrow \omega_{\text {ref }}=\sqrt{2 \beta \max q}$

ii) if $-q_{f}<q<0 \longrightarrow \omega_{\text {ref }}=-\sqrt{2 \beta_{\max }(-q)}$

iii) if $\quad q_{f}<q<q_{\max } \longrightarrow \omega_{\text {ref }}=\omega_{\max }$

iv) if $-q_{\max }<q<-q_{f} \longrightarrow \omega_{\text {ref }}=-\omega_{\max }$

where:

$q=$ actual error position.

$q_{f}=$ position error where the deceleration process starts.

$\beta_{\max }=$ digital value that represents the maximum rate of deceleration

$q_{\max }=$ maximum error position (32767).

$=$ maximum position command.

These rules are shown graphically in Fig. 5.

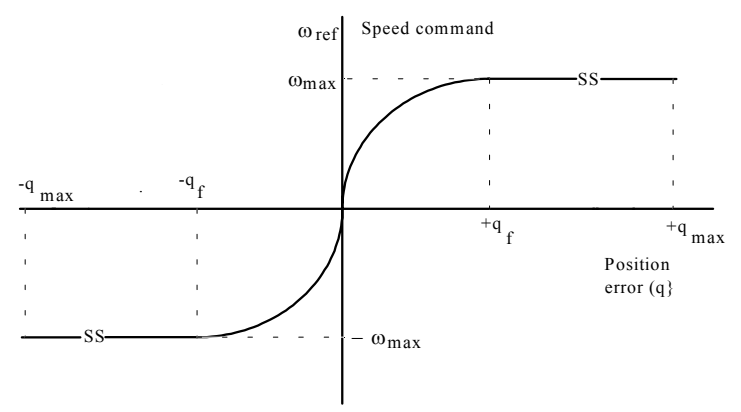

Fig. 5. Speed commands in function of the error position

\section{Position and Speed Measurement}

The rotor position is measured by capturing, by means of the microcontroller's High Speed Input Unit, the pulse trains from an encoder mounted on the motor's shaft. Input HSI.0 captures the pulse train from the encoder's channel A, HSI.1 captures the pulse train from the encoder's channel B, and HSI.2 captures the pulse train from the encoder's index. Both channels A and B are captured in order to detect the direction of the movement.

Input HSI.0 and HSI.1 are programmed to 
generate an interrupt at both positive and negative edges of the signals from channels $\mathrm{A}$ and $\mathrm{B}$. In this way, 1024 transitions per revolution can be detected, using a 256-slit encoder. Input HSI.2 generates an interrupt only at the negative edge of the index signal.

This signal is utilized to make an adjustment of the rotor position at the end of each revolution. This procedure corrects possible errors in the detection of the signals from channels $\mathrm{A}$ and $\mathrm{B}$.

To detect the direction of the movement, an algorithm that depends on the states of the signals from channels $\mathrm{A}$ and $\mathrm{B}$ when a transition occurs is applied in the corresponding interrupt service routine. This algorithm is shown in Table 1.

Table 1

Algorithm to Detect the Direction of the Movement

\begin{tabular}{|l|cr|lrl|ll||}
\hline Direction & \multicolumn{2}{|c|}{$\begin{array}{c}\text { Channel } \\
\text { A } \\
\text { interrupt }\end{array}$} & $\begin{array}{c}\text { Channel } \\
\text { B } \\
\text { interrupt }\end{array}$ & $\begin{array}{c}\text { Index } \\
\text { interrupt }\end{array}$ \\
\hline \hline & A & B & A & B & A & B \\
\hline Clockwise & 1 & 0 & 1 & 1 & 1 & 0 \\
\hline Clockwise & 0 & 1 & 0 & 0 & & \\
\hline counter clockwise & 1 & 1 & 1 & 0 & 0 & 1 \\
\hline counter clockwise & 0 & 0 & 0 & 1 & & \\
\hline
\end{tabular}

The speed is calculated by using the arrival times of the pulse trains from the encoder. The pulse arrival times are captured by the high speed input unit of the microcontroller. To obtain a precise measurement of the speed rotor, two different algorithms are applied:

For high speeds, the number of pulses that occur during the sample period $(300 \mu \mathrm{s})$ are counted. When the sample period is completed, the microcontroller waits for the next pulse. After this pulse arrives, the microcontroller reads the additional time that elapsed from the end of the sample period to the arrival of the next pulse. In this case the speed $\omega$ is calculated by using (6).

$$
\omega=\frac{\text { total number of pulses }}{\text { total time elapsed }[\mathrm{s}]}
$$

For low speeds, the time elapsed between two consecutive pulses is measured and the speed is calculated by using (7).

$$
\omega=\frac{1 \text { pulse }}{\text { time between two consecutive pulses [s] }}
$$

\section{Design of the Speed Controller}

To control the speed, a digital IntegralProportional (IP) controller was designed [6]. Fig. 6 shows the speed control loop, in analog form.

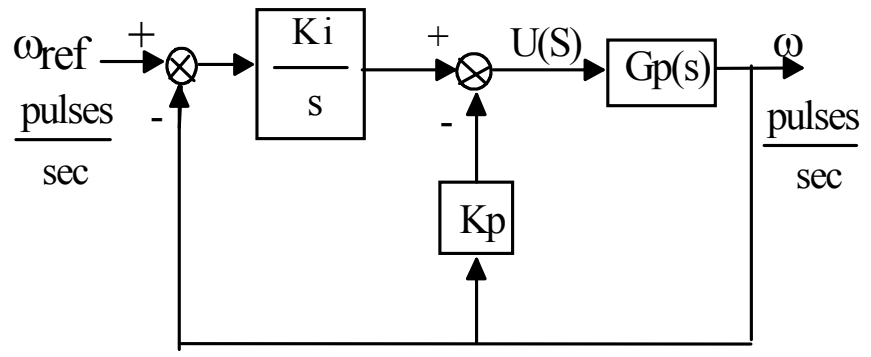

Fig. 6. Speed control loop

In this diagram, $G p(s)$ includes the $\mathrm{D} / \mathrm{A}$ converter, the PWM circuit, the dc-dc converter, the dc motor, and the mechanical system. To design the gains of the IP controller, it is required to know the mathematical model of $\mathrm{Gp}(\mathrm{s})$. A description of the method applied for the estimation of $\mathrm{Gp}(\mathrm{s})$ follows.

\subsection{Estimation of the System Model}

Since the PWM controller uses high switching frequency $(15 \mathrm{KHz})$, a lineal approximation of $\mathrm{Gp}(\mathrm{s})$ can be used. Usually, the transfer function of a dc motor, with voltage as the input and speed as the output, is approximated using a second order model. However, since the electrical time constant of the motor is much lower than its mechanical time constant, the electrical time constant can be assumed to be negligible and, hence, to design the speed controller, it is a reasonable approximation to represent the dc motor by means of a first order model. Therefore, the block $\mathrm{Gp}(\mathrm{s})$ can be approximated by using (8). 


$$
G p(s)=\frac{\omega(s)}{U(s)}=\frac{A}{\tau s+1}
$$

where:

$\omega=$ angular speed in pulses/s

$\mathrm{U}=$ speed command, in digital form, sent by the microcontroller

$\mathrm{A}=\mathrm{CD}$ gain (final value of the step response)

$\tau=$ Time constant, in seconds.

Using this approximation, the model of each servomechanism was estimated by applying a step input (digital command $\mathrm{u}=1629=$ constant) to each block $\mathrm{Gp}(\mathrm{s})$, recording the corresponding speed output, and using two points of this response and (9) to solve for A and $\tau$. Equation (9) is the step response of (8).

$$
\omega(t)=A u\left(1-e^{-t / \tau}\right)
$$

\subsection{Design of the Controller Gains}

The closed loop transfer function of the speed control system shown in Fig. 6, with the first order approximation for $\mathrm{Gp}(\mathrm{s})$, is given by (10).

$$
G(s)=\frac{\frac{K_{i} A}{\tau}}{s^{2}+\left(\frac{1+K_{p}^{A}}{\tau}\right) s+\frac{K_{i} A}{\tau}}
$$

Transforming (10) into the digital form we obtain:

$G(z)=\frac{A_{1} K_{i d}}{z^{2}+\left[A_{1}\left(K_{p d}+K_{i d}\right)-R-1\right] z+R-A_{1} K_{p d}}$

where:

$K_{i}=$ analog integral gain

$K_{p}=$ analog proportional gain

$R=R(T)=e^{-T / \tau}$

$T=$ sample period

$A_{1}=A_{1}(T)=A[1-R(T)]$

$K_{i d}=K_{i} T=$ digital integral gain

$K_{p d}=K_{p}-\frac{1}{2} K_{i d}=$ digital proportional gain
Comparing (10) with the transfer function of a generalized second order system:

$$
G(s)=\frac{\omega^{2}{ }_{n}}{s^{2}+2 \zeta \omega_{n} s+\omega^{2}{ }_{n}}
$$

where:

$$
\begin{aligned}
& \zeta=\text { damping ratio } \\
& \omega_{n}=\text { undamped natural frequency }
\end{aligned}
$$

We get:

$$
\begin{aligned}
K_{i} & =\frac{\omega^{2}{ }_{n} \tau}{A} \\
K_{p} & =\frac{2 \zeta \omega_{n} \tau-1}{A}
\end{aligned}
$$

In this way, the analog gains $K_{i}$ and $\mathrm{K}_{\mathrm{p}}$, and therefore the digital controller gains $K_{i d}$ and $\mathrm{K}_{\mathrm{pd}}$, can be calculated by selecting the values of $\omega_{n}$ and $\zeta$ that give the desired behavior of the closed loop control system. To select these values, the equations that define the maximum overshoot and the settling time for an underdamped second-order system were used.

\section{Experimental Results}

After the design and construction a testing of the manipulator was carried out. First, the individual hardware components were tested. The testing was performed in the following sequence: PWM signal generator circuits, cd-cd power electronic converters, and microcontroller interfaces. After the hardware testing, the software modules were tested in the following sequence: position and speed control of each servomechanism, the master control software, and the PC-microcontrollers communication software. Finally, the testing included the global evaluation of the manipulator.

A good control of each servomechanism is the key factor to evaluate how useful a manipulator will be. For this reason, special attention was paid to the evaluation of the position and speed controllers designed for each servomechanism. Fig. 7 shows the response of a servomechanism to a position command of 2000 pulses. The plot shows a good servomechanism response with minimum overshoot. 
Fig. 8 shows the corresponding speed controller response. It can be seen that the servomechanism speed response adjusts to the speed profile shown in Fig. 4 and to the algorithm given by (2), (3), (4), and (5).

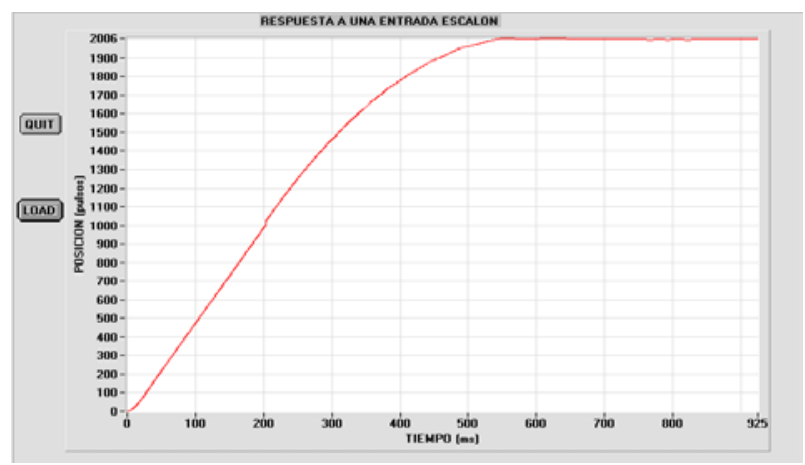

Fig. 7. Response of a servomechanism to a step position command

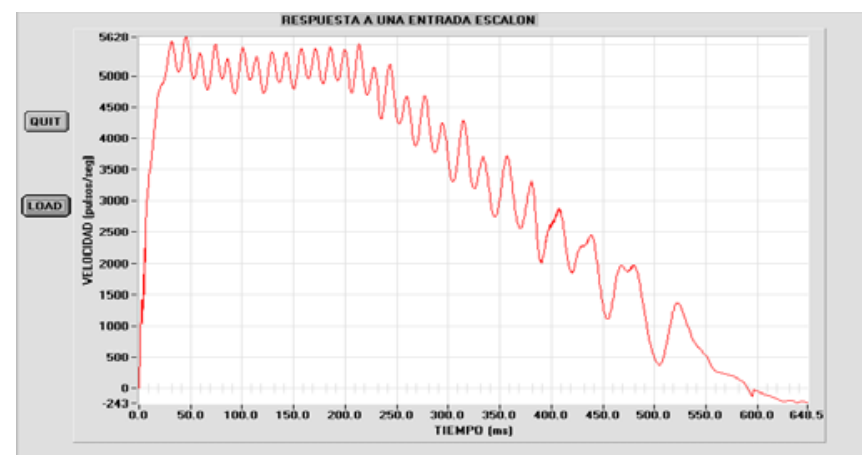

Fig. 8. Speed response of a servomechanism

To test the manipulator in the printed-circuitboard driller system, several Orcad files were loaded and run using the graphical user interface. The boards were drilled with a very good accuracy. The manipulator uses gear mechanisms with a 75 to 1 coupling ratio, feed screws with pitch of $0.3 \mathrm{~cm} /$ turn, and encoders that give 1024 transitions/turn. In this way, the manipulator has a resolution of $3.9 \times 10^{-8} \mathrm{~m}$. printed-circuit-board driller system. The details of the hardware and software designed for the manipulator were presented. The design includes a special algorithm to control the position of each servomechanism. In this algorithm the controllers generate speed commands that depend on the error position. The algorithms used to measure position and speed resulted with a very good accuracy. The paper described the design of a digital integral-proportional speed controller, including the method applied to estimate the model of each servomechanism. The estimated model includes the D/A converter, the PWM circuit, the dc-dc electronic converter, the dc motor, and the mechanical system. The testing performed on each servomechanism showed the effectiveness of the design presented in this paper.

References

[1] K. S. Fu, R. C. González , C. S. G. Lee, Robotica Control, Detección, Visión e Inteligencia, Mc Graw Hill, 1989

[2] Mohan/Undeland/Robbins, Power Electronics Converts, Applications and Design, John Wiley \& Sons.

[3] 16 Bit Embedded Controllers, Intel 1990.

[5] Edmundo Barrera Cardiel, An HVDC Transmission System Model for a Physically Based Power System Laboratory, doctoral dissertation, The University of Texas at Arlington, USA, 1991.

[6] Takashi Kenjo, Power Electronics for the Microprocessors Age, Oxford Science Publications, 1994.

[7] Katsuhiko Ogata, Modern Control Engineering, Prentice Hall.

\section{Conclusions}

The paper presented the design and construction of a 3-axis manipulator and its application to a 